\title{
Oestradiol metabolism and androgen receptor genotypes are associated with right ventricular function
}

\author{
Corey E. Ventetuolo ${ }^{1}$, Nandita Mitra ${ }^{2}$, Fei Wan², Ani Manichaikul ${ }^{3}$, \\ R. Graham Barr ${ }^{4}$, Craig Johnson ${ }^{5}$, David A. Bluemke ${ }^{6}$, Joao A.C. Lima ${ }^{7}$, \\ Hari Tandri ${ }^{7}$, Pamela Ouyang ${ }^{7}$ and Steven M. Kawut ${ }^{2,8,9}$
}

\begin{abstract}
Affiliations: ${ }^{1}$ Depts of Medicine and Health Services, Policy and Practice, Alpert Medical School of Brown University, Providence, RI, USA. ${ }^{2}$ Dept of Biostatistics and Epidemiology, University of Pennsylvania, Philadelphia, PA, USA. ${ }^{3}$ Center for Public Health Genomics, Department of Public Health Sciences, University of Virginia, Charlottesville, VA, USA. 'Dept of Medicine, College of Physicians and Surgeons, Columbia University, New York, NY, USA. ${ }^{5}$ Dept of Biostatistics, University of Washington, Seattle, WA, USA. ${ }^{6}$ Radiology and Imaging Sciences, National Institutes of Health/Clinical Center, National Institute for Biomedical Imaging and Bioengineering, Bethesda, MD, USA. ${ }^{7}$ Dept of Medicine, Johns Hopkins School of Medicine, Baltimore, MD, USA. ${ }^{8}$ Dept of Medicine, University of Pennsylvania, Philadelphia, PA, USA. ${ }^{9}$ Penn Cardiovascular Institute, Perelman School of Medicine, University of Pennsylvania, Philadelphia, PA, USA.
\end{abstract}

Correspondence: Corey E. Ventetuolo, Division of Pulmonary, Critical Care and Sleep, Rhode Island Hospital, APC 747, 593 Eddy Street, Providence, RI 02903, USA. E-mail: corey_ventetuololabrown.edu

ABSTRACT Sex hormones are linked to right ventricular (RV) function, but the relationship between genetic variation in these pathways and RV function is unknown.

We performed a cross-sectional study of 2761 genotyped adults without cardiovascular disease. The relationships between RV measures and single nucleotide polymorphisms (SNPs) in 10 candidate genes were assessed. Urinary oestradiol (E2) metabolites produced by cytochrome P4501B1 (CYP1B1) and serum testosterone were measured in women and men respectively.

In African-American (AA) women, the CYP1B1 SNP rs162561 was associated with RV ejection fraction (RVEF), such that each copy of the A allele was associated with a $2.0 \%$ increase in RVEF. Haplotype analysis revealed associations with RVEF in AA (global $\mathrm{p}<7.2 \times 10^{-6}$ ) and white (global $\mathrm{p}=0.05$ ) women. In white subjects, higher E2 metabolite levels were associated with significantly higher RVEF. In men, androgen receptors SNPs (rs1337080; rs5918764) were significantly associated with all RV measures and modified the relationship between testosterone and RVEF.

Genetic variation in E2 metabolism and androgen signalling was associated with RV morphology in a sex-specific manner. The CYP1B1 SNP identified is in tight linkage disequilibrium with SNPs associated with pulmonary hypertension and oncogenesis, suggesting these pathways may underpin sexual dimorphism in RV failure.

@ERSpublications

Oestradiol metabolism and androgen receptor genotypes are associated with RV morphology in a sex-specific manner http://ow.ly/UbUJ3

For editorial comment see Eur Respir J 2016; 47: 390-393 [DOI: 10.1183/13993003.01999-2015].

This article has supplementary material available from erj.ersjournals.com

Received: July 062015 | Accepted after revision: Oct 092015 | First published online: Dec 032015

Support statement: This work was completed with support from the American Heart Association 11FTF7400032, National Institutes of Health P20GM103652 and K24 HL103844. MESA and the MESA SHARe project are conducted and supported by the National Heart, Lung, and Blood Institute (NHLBI) in collaboration with MESA investigators. Support for MESA is provided by contracts R01-HL086719, N01-HC-95159, N01-HC-95160, N01-HC-95161, N01-HC-95162, N01-HC-95163, N01-HC-95164, N01-HC-95165, N01-HC-95166, N01-HC-95167, N01-HC-95168, N01-HC-95169, UL1-TR-001079, and UL1-TR-000040. Funding for SHARe genotyping was provided by NHLBI Contract N02-HL-64278. Funding information for this article has been deposited with FundRef.

Conflict of interest: Disclosures can be found alongside the online version of this article at erj.ersjournals.com

The content of this work is not subject to copyright. Design and branding are copyright @ERS 2016. 


\section{Introduction}

Idiopathic and heritable pulmonary arterial hypertension (PAH) occur more commonly in women, and extensive animal and human data support a role for sex hormones in disease pathogenesis and outcomes [1]. Genetic variation in sex hormone pathways (specifically the genes responsible for oestradiol (E2) metabolism cytochrome P450 lb1 (CYP1B1), aromatisation, and receptor signalling) have been linked to several PAH subtypes [2-4]. In animal and human pulmonary arterioles, there is increased expression of both CYP1B1 and aromatase and these pathways have been proposed as possible therapeutic targets $[5,6]$.

Beyond PAH, right ventricular (RV) function is an important cause of morbidity and mortality in highly prevalent cardiopulmonary diseases, but genetic determinants of the RV response to chronic heart and lung derangements are entirely unknown [7-9]. Cytochrome P450 enzymes are preferentially expressed in the RV compared to the left ventricle (LV) in human hearts, with increased sex hormone metabolism and steroid receptor (specifically androgen receptor) expression in models of cardiac hypertrophy, but these relationships have not been explored in vivo $[10,11]$.

Recent epidemiological data have shown that women with pulmonary hypertension of varied aetiologies have better survival than men $[12,13]$. This may be due to sex-mediated differences in disease burden at baseline, response to therapy, and/or RV adaptation, a major determinant of outcome in chronic heart and lung disease, including PAH [14-17]. Race has also been linked to pulmonary hypertension prevalence and outcomes, with registries reporting female:male ratios as high as 4-5:1 in African-American subjects compared with 2:1 for white subjects, but higher rates of death among African-American subjects [18-21]. Sex- or gene-based variations in E2 metabolism resulting in metabolites with different mitogenic potential and altered sex hormone receptor signalling with differential effects on the RV could explain these findings.

We have previously shown that sex, sex hormones and race are associated with RV measures in adults without clinical cardiovascular disease, with female sex, higher levels of oestrogen/lower levels of androgens, and white race associated with more favourable RV morphology [14, 22]. In this study, we sought to determine whether genetic variation in sex hormone pathways is associated with RV ejection fraction (RVEF), end-diastolic mass and end-diastolic volume (RVEDV) in a sex- and race-specific manner in a population-based, multi-ethnic cohort. We also determined whether single nucleotide polymorphisms (SNPs) of interest were associated with sex hormones (urinary E2 metabolites and serum testosterone) and if these levels were integral to genotype-phenotype associations. We hypothesised that variation in the CYP1B1 gene would be associated with RV function and that this relationship would be mediated by the E2 metabolites 2-hydroxyestrone (2-OHE) and 16 $\alpha$-hydroxysterone (16-OHE1) in women and that androgen processing and signalling would be associated with RV function in men via serum testosterone levels.

\section{Methods}

Study sample

The Multi-Ethnic Study of Atherosclerosis (MESA) is a multicentre prospective cohort study to investigate the prevalence, correlates and progression of subclinical cardiovascular disease in an ethnically enriched population from six US communities [23]. In 2000-2002, MESA recruited 6814 subjects aged $45-84$ years old and excluded those with clinical cardiovascular disease, weight $>136 \mathrm{~kg}(>300 \mathrm{lbs})$, pregnancy or impediment to long-term participation. The protocols of MESA and studies described herein were approved by the Institutional Review Boards of all collaborating institutions and the National Heart Lung and Blood Institute.

\section{Cardiac magnetic resonance imaging measures}

The MESA-Right Ventricle Study measured RV morphology in participants eligible for cardiac magnetic resonance imaging (MRI). The cardiac MRI protocol has been described elsewhere with methods of interpretation for left and right ventricular parameters [22]. The intra-class correlation coefficients (ICCs) from 229 random, blinded intra-reader re-reads of these scans were $0.93,0.93$ and 0.99 for RVEF, RV mass and RVEDV, respectively. The ICCs from inter-reader reads of 240 scans for RVEF, RV mass, and RVEDV were $0.75,0.87$, and 0.96 , respectively.

\section{Genotyping}

Genomic DNA was extracted from whole blood or packed cells stored at $-70^{\circ} \mathrm{C}$ using a modified salt precipitation method (Puregene; Qiagen Instrument Service, Germantown, MD, USA). The study sample was genotyped for SNPs in sex hormone pathways of interest using the IBC chip (ITMAT-Broad-CARe, Illumina, San Diego, CA, USA), a $50 \mathrm{~K}$ SNP array designed to capture relevant loci in cardiovascular, metabolic, and inflammatory disease processes [24]. Ancestry informative markers and admixture SNPs were also included to account for population stratification. We selected 463 SNPs in 10 candidate genes a priori for this study, including the oestrogen receptor 1 and 2, oestrogen receptor-related $\gamma$, oestrogen related receptor $\alpha$, breast cancer-associated gene 3, CYP1B1, cytochrome P450 1A1, catechol-o-methyltransferase, aromatase and androgen receptor genes (table 1). 


\begin{tabular}{lccc}
$\begin{array}{l}\text { TABLE } 1 \text { Sex hormone pathways of interest and candidate genes } \\
\text { Pathway }\end{array}$ & Gene & Chromosome & SNP \\
\hline Oestrogen signalling & Oestrogen receptor 1 (ESR1) & 6 & 285 \\
& Oestrogen receptor 2 (ESR2) & 14 & 43 \\
& Oestrogen receptor related gamma (ESRRG) & 1 & 5 \\
& Oestrogen related receptor alpha (ESSRA) & 11 & 7 \\
Oestrogen metabolism & Breast cancer-associated gene 3 (BCAS3) & 17 & 2 \\
& Cytochrome P450 1B1 (CYP1B1) & 2 & 13 \\
Androgen to oestrogen conversion & Cytochrome P450 1A1 (CYP1A1) & 15 & 17 \\
Androgen signalling & Catechol-O-methyltransferase (COMT) & 22 & 21 \\
& Aromatase (CYP19A1) & 15 & 45 \\
& Androgen receptor (AR) & $X$ & 25
\end{tabular}

SNP: single nucleotide polymorphism.

Urinary oestradiol metabolites and serum sex hormone levels

Fasting, casual spot urine samples were collected during the MESA baseline examination, at the same time as cardiac MRI and collection of blood samples. $0.230 \mathrm{~mL}$ of acetic acid was added before storage at $-80^{\circ}$ C. Urinary E2 metabolites were measured at the Laboratory for Clinical Biochemistry Research at the University of Vermont (Burlington, VT, USA) using a monoclonal antibody-based enzyme assay (Estramet 2/16, Immuna Care, Tampa, FL, USA). Urine was diluted 1:4 with the manufacturer's diluent and assays were run in duplicate, with intra-assay coefficients of variation of $8 \%$ for 2 -OHE and $9 \%$ for 16 -OHE1. Urine creatinine levels were also measured and adjusted for in multivariate analyses, as described below.

Fasting morning blood samples were drawn and stored using standardised procedures. Total testosterone was measured directly with radioimmunoassay kits (Diagnostic Products Corporation, Los Angeles, CA, USA). Assay quality control has been described elsewhere; the intra-assay coefficient of variation for testosterone has been reported to be $12 \%$ [25].

\section{Other covariates}

Race/ethnicity was self-reported during the baseline exam. For women, self-report was used to determine pre-, peri-, or post-menopausal status. Medication use, including current hormone therapy, was ascertained by medication inventory [26]. Standard questionnaires were used to obtain smoking status and level of education. Height was measured to the nearest $0.1 \mathrm{~cm}$ with the participant in stocking feet, and weight was measured to the nearest pound with the participant in light clothing using a balanced scale. Waist circumference was measured at the level of the umbilicus with the participant breathing normally using a Gulick II anthropometric tape measure. Resting blood pressure was measured using the Dinamap Monitor PRO 100 (Critikon, Tampa, FL, USA) automated oscillometric device. Hypertension was defined as systolic blood pressure $\geqslant 140 \mathrm{mmHg}$, diastolic blood pressure $\geqslant 90 \mathrm{mmHg}$ or current use of anti-hypertension medication. Presence of diabetes mellitus was based on self-reported physician diagnosis or a fasting glucose value $\geqslant 126 \mathrm{mg} \cdot \mathrm{dL}^{-1}$, the latter measured by rate reflectance spectrophotometry (Johnson \& Johnson Clinical Diagnostics, Inc., Rochester, NY, USA). Fasting glucose of $100-125 \mathrm{mg} \cdot \mathrm{dL}^{-1}$ was considered impaired fasting glucose.

\section{Statistical analysis}

Continuous variables were expressed as mean \pm SD and categorical variables were expressed as percentages. The associations between the level of each continuous RV measure (RVEF, RV mass and RVEDV) and SNPs of interest were assessed using multivariable linear regression models. An underlying additive genetic model was assumed in order to obtain a per-allele effect estimate. Analyses for RV outcomes (RVEF, RV mass and RVEDV) were first stratified by sex and then self-identified race/ethnicity (white, AfricanAmerican, Hispanic or Chinese-American). All analysed SNPs of interest met a minor allele frequency cut-off of $>0.05$ and were tested for Hardy-Weinberg equilibrium ( $p$-value cut-off of 0.001 ). Risk allele frequency by sex and race/ethnicity for SNPs of interest is presented in table E1 in the online supplemental material. The sex hormone genotype-RV phenotype models were adjusted for age, height, weight, study site and the top three principal components based on ancestry informative markers (to adjust for residual population stratification). Analyses were then adjusted for respective LV measures (e.g., models for RVEF were adjusted for LV ejection fraction). Models were adjusted for hormone therapy use in women. All p-values were adjusted for multiple comparisons (number of SNPs within each specific gene of interest) using the Bonferroni adjustment. Meta-analyses were also conducted across race/ethnicity using METAL for 
each RV outcome (RVEF, mass, and RVEDV). When more than one SNP was associated with RV function in a given gene, or when significant SNPs were noted in genes previously described in disease states of interest (i.e., PAH and oncogenesis), we assessed haplotype structure (Haploview 4.0) and conducted haplotype analyses (haplo.stats in R, R Foundation for Statistical Computing, Vienna, Austria).

Urinary E2 metabolites were natural log-transformed. As genotype-phenotype analyses were stratified by race/ethnicity (and further adjusted for residual population stratification), we first assessed whether there was effect modification between E2 metabolites and race/ethnicity, and conducted secondary analyses stratified by race/ethnicity when the interaction was found to be significant $(p<0.05)$. The relationship between $\ln (2-\mathrm{OHE}), \ln (16-\mathrm{OHE} 1)$, and the $\ln (2-\mathrm{OHE} / 16-\mathrm{OHE} 1)$ ratio and RVEF, RV mass and RVEDV was assessed using multivariable linear regression. Analyses were adjusted for age, race/ethnicity, height, weight, waist circumference, smoking (pack-years and smoking status), diabetes mellitus, impaired glucose tolerance, systemic hypertension, urine creatinine levels, total cholesterol and high-density lipoprotein levels, education level, hormone therapy use and respective LV parameters.

Last, to investigate whether E2 metabolites were mediators in the CYP1B1-RV relationship, $\ln (2-\mathrm{OHE}), \ln$ $\left(16-\mathrm{OHE}_{1}\right)$, and the $\ln \left(2-\mathrm{OHE} / 16-\mathrm{OHE}_{1}\right)$ ratio were sequentially added to the genotype-phenotype multivariable model in order to assess whether the addition of a metabolite attenuated the estimated coefficient for the SNP of interest [27]. In men, we tested whether there were interactions between SNPs in the androgen receptor and testosterone levels and RV function. If there was a $>10 \%$ change in the estimated coefficient between a given SNP and RV measure with the addition of hormone levels to the model, modified Sobel tests were used to assess whether the degree of mediation was statistically significant [28]. Statistical significance was defined as $\mathrm{p}<0.05$. These analyses were performed using SAS v.9.2 (SAS Institute, Inc, Cary, NC) and Stata v. 10.0 (StataCorp, College Station, TX, USA).

\section{Results}

A total of 2761 participants had available SNP genotypes in the sex hormone pathways of interest and biological samples (figure 1). The cohort was $47 \%$ female, of whom $44 \%$ were white, $24 \%$ were AfricanAmerican, 18\% were Hispanic and 14\% were Chinese-American (table 2). Men and women had similar characteristics, although women tended to be never-smokers and had higher RVEF and lower RV mass and RVEDV, as has been previously described [22]. Among women, 97\% were post-menopausal and 34\% were using hormone therapy at the time of the MESA baseline examination (in 2000-2002).

\section{CYP1B1 genotype and RV phenotype in women}

A single SNP in the CYP1B1 gene (rs162561) was associated with RVEF in African-American women, such that for each additional " $\mathrm{A}$ " allele there was a 2.0\% increase in RVEF after adjustment for age, height, weight, study site, top three principal components and hormone therapy (Bonferroni-corrected $\mathrm{p}=0.02$ ) (table 3). This relationship was slightly attenuated by the addition of LV ejection fraction to the model. SNP rs162561 was in tight linkage disequilibrium with rs1800440 ( $D=0.76)$, which has been described to increase PAH penetrance in bone morphogenetic protein receptor type 2 female mutation carriers, and with rs1056836 $(\mathrm{D}=0.96)$, which has been associated with increased breast and lung cancer risk in some

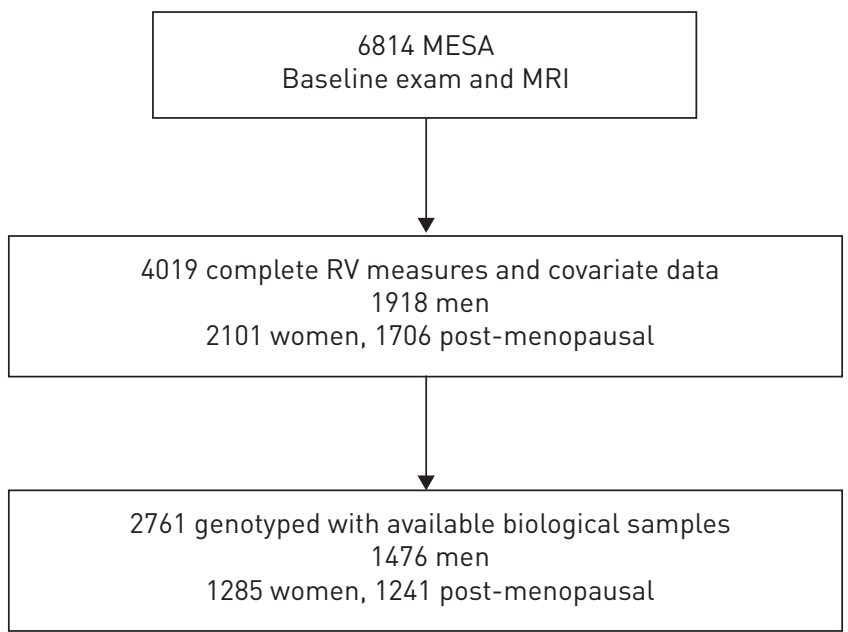

FIGURE 1 Study sample. MESA: Multi-ethnic Study of Atherosclerosis; MRI: magnetic resonance imaging; RV: right ventricle. 
TABLE 2 Participant characteristics, by sex

\begin{tabular}{|c|c|c|}
\hline & \multicolumn{2}{|c|}{ Sex } \\
\hline & Men & Women \\
\hline Participants n & 1476 & 1285 \\
\hline \multicolumn{3}{|l|}{ Demographics } \\
\hline Age years & $62.1 \pm 10.1$ & $64.3 \pm 9.1$ \\
\hline \multicolumn{3}{|l|}{ Race/ethnicity } \\
\hline White & 43 & 44 \\
\hline African-American & 22 & 24 \\
\hline Hispanic & 21 & 18 \\
\hline Chinese-American & 14 & 14 \\
\hline \multicolumn{3}{|l|}{ Education } \\
\hline$<$ High school & 15 & 18 \\
\hline High school & 15 & 21 \\
\hline <College (>high school) & 26 & 31 \\
\hline$\geqslant$ College & 44 & 30 \\
\hline \multicolumn{3}{|l|}{ Anthropometrics } \\
\hline Height $\mathrm{cm}$ & $173.4 \pm 7.7$ & $159.8 \pm 7.2$ \\
\hline Weight kg & $82.6 \pm 14.9$ & $71.7 \pm 15.5$ \\
\hline Body mass index $\mathrm{kg} \cdot \mathrm{m}^{-2}$ & $27.4 \pm 4.1$ & $28.0 \pm 5.5$ \\
\hline \multicolumn{3}{|l|}{ Disease prevalence } \\
\hline Hypertension & 42 & 49 \\
\hline Systolic blood pressure $\mathrm{mmHg}$ & $125.2 \pm 19.0$ & $128.5 \pm 23.5$ \\
\hline Diastolic blood pressure $\mathrm{mmHg}$ & $74.7 \pm 9.3$ & $69.3 \pm 10.4$ \\
\hline \multicolumn{3}{|l|}{ Diabetes mellitus } \\
\hline None & 70 & 76 \\
\hline Impaired fasting glucose & 17 & 13 \\
\hline Untreated diabetes & 3 & 2 \\
\hline Treated diabetes & 10 & 9 \\
\hline Total cholesterol $\mathrm{mg} \cdot \mathrm{dL}^{-1}$ & $187.6 \pm 33.9$ & $202.2 \pm 35.7$ \\
\hline High-density lipoprotein $\mathrm{mg} \cdot \mathrm{dL}^{-1}$ & $44.9 \pm 11.7$ & $57.0 \pm 15.6$ \\
\hline \multicolumn{3}{|l|}{ Smoking status } \\
\hline Never-smoker & 43 & 60 \\
\hline Former smoker & 44 & 29 \\
\hline Current smoker & 13 & 11 \\
\hline Pack-years among ever-smokers & $14.1 \pm 29.4$ & $8.6 \pm 18.2$ \\
\hline \multicolumn{3}{|c|}{ Menopausal status and hormone therapy use } \\
\hline Pre-menopausal & & 3 \\
\hline Post-menopausal & & 97 \\
\hline Hormone therapy & & 34 \\
\hline \multicolumn{3}{|l|}{ Urinary and serum sex hormone levels } \\
\hline \multicolumn{3}{|l|}{ Hormone therapy user } \\
\hline Oestradiol $\mathrm{nmol} \cdot \mathrm{L}^{-1}$ & & $0.3 \pm 0.2$ \\
\hline 2-hydroxyestrone $\mathrm{ng} \cdot \mathrm{mL}^{-1}$ & & $15.8 \pm 8.8$ \\
\hline $16 \alpha$-hydroxysterone $\mathrm{ng} \cdot \mathrm{mL}^{-1}$ & & $9.2 \pm 6.1$ \\
\hline \multicolumn{3}{|l|}{ Hormone therapy non-user } \\
\hline Oestradiol $\mathrm{nmol} \cdot \mathrm{L}^{-1}$ & & $0.1 \pm 0.1$ \\
\hline 2-hydroxyestrone $\mathrm{ng} \cdot \mathrm{mL}^{-1}$ & & $8.9 \pm 5.9$ \\
\hline $16 \alpha$-hydroxysterone $\mathrm{ng} \cdot \mathrm{mL}^{-1}$ & & $5.2 \pm 3.5$ \\
\hline Total testosterone $\mathrm{nmol} \cdot \mathrm{L}^{-1}$ & $15.0 \pm 5.5$ & \\
\hline Bioavailable testosterone $\mathrm{nmol} \cdot \mathrm{L}^{-1}$ & $5.5 \pm 2.2$ & \\
\hline \multicolumn{3}{|l|}{ RV measures } \\
\hline RV ejection fraction \% & $68.4 \pm 6.3$ & $72.7 \pm 5.9$ \\
\hline $\mathrm{RV}$ mass $\mathrm{g}$ & $22.9 \pm 4.4$ & $18.9 \pm 3.5$ \\
\hline $\mathrm{RV}$ end-diastolic volume $\mathrm{mL}$ & $140.0 \pm 29.4$ & $107.3 \pm 22.2$ \\
\hline
\end{tabular}

Data are presented as mean \pm SD or $\%$, unless otherwise stated. RV: right ventricle.

studies [4, 29-32]. Haplotype analysis containing these three SNPs (rs162561, rs1800440 and rs1056836) revealed significant associations with RVEF in African-American subjects (global $\mathrm{p}<7.2 \times 10^{-6}$ ), whites (global $\mathrm{p}=0.05$ ), but not Hispanics (global $\mathrm{p}=0.13$ ) or Chinese-Americans $(\mathrm{p}=0.24)$ although the sample sizes of these latter groups were limited (figure 2). 
TABLE 3 Selected additive multivariable linear regression models for CYP1B1 SNP and RVEF in women and androgen receptor SNPs and RV measures in men

\begin{tabular}{|c|c|c|c|c|c|c|c|}
\hline Sex & Race/ethnicity & Gene & SNP & Risk allele & RV measures & $\boldsymbol{\beta} \pm \mathrm{SE}$ & Bonferroni p-value \\
\hline Women & & & & & $\mathrm{RVEF}^{\#, \uparrow}$ & $1.7 \pm 0.6$ & 0.08 \\
\hline \multirow{7}{*}{ Men } & & & & & RV mass ${ }^{\pi}$ & $0.9 \pm 0.3$ & 0.02 \\
\hline & & & & & RVEDV & $5.7 \pm 1.8$ & 0.05 \\
\hline & & & & & RVEDV? & $3.0 \pm 1.4$ & 0.75 \\
\hline & & & & & RVEDV & $5.7 \pm 1.8$ & 0.05 \\
\hline & & & & & RVEDVף & $3.0 \pm 1.4$ & 0.75 \\
\hline & Meta-analysis & AR & rs1337080 & G & RV mass & $1.0 \pm 0.3$ & 0.03 \\
\hline & & & & & RV mass ${ }^{\natural}$ & $0.9 \pm 0.3$ & 0.03 \\
\hline
\end{tabular}

All adjusted for age, height, weight, study site, and top 3 principal components. " : adjusted for hormone therapy. ": adjusted for respective left ventricular measure. CYP1B1: cytochrome P450 1B1; AR: androgen receptor; SNP: single nucleotide polymorphism; RVEF: right ventricular ejection fraction; RVEDV: right ventricular end-diastolic volume.

Urinary $E 2$ metabolites and RV measures in women

Women using hormone therapy had higher levels of 2- and $16 \alpha$-hydroxyestrone (and higher levels of oestradiol), as expected (table 1). We found statistically significant interactions between race and $\ln$ (2-OHE) and $\ln \left(16-\mathrm{OHE}_{1}\right)$ for RVEF $(\mathrm{p}<0.01$ for both metabolites), but not RV mass or RVEDV. In whites, higher $\ln (2-\mathrm{OHE})$ and $\ln \left(16-\mathrm{OHE}_{1}\right)$ levels were associated with significantly higher RVEF after adjustment for multiple potential confounders including urine creatinine levels, hormone therapy and left ventricular ejection fraction (table 4). Conversely, higher $\ln \left(16-\mathrm{OHE}_{1}\right)$ levels were associated with lower RVEF in Hispanics. There were no such associations between E2 metabolites and RVEF in AfricanAmerican or Chinese-American subjects or between E2 metabolites and RV mass or RVEDV in the total cohort. Urinary E2 metabolites did not meet our threshold for mediation of the CYP1B1 genotype-RV phenotype relationships (data not shown).

\section{Androgen receptor genotype and $R V$ phenotype in men}

Two SNPs in the androgen receptor gene (rs1337080 and rs5918764) were associated with multiple RV measures in white men (table 3). For both SNPs, each additional risk allele was associated with approximately a $1 \mathrm{~g}$ increase in RV mass and a $6 \mathrm{~mL}$ higher RVEDV in multivariable analysis. Some of these relationships were weakened by the addition of respective LV measures to the model. Meta-analysis across race/ethnicity revealed that each copy of the $G$ allele in rs1337080 was associated with approximately a $1 \mathrm{~g}$ increase in RV mass, even after adjustment for the LV ( $p=0.03$ ). The two SNPs in the androgen receptor gene were tightly linked $(\mathrm{D}=0.90)$.

\section{Effect modification by the androgen receptor genotype on the testosterone-RV phenotype relationship in men}

We have previously shown that higher levels of serum testosterone were associated with greater RV mass and larger volumes in men from this cohort [14]. There were significant interactions between testosterone levels and the androgen receptor SNPs and RVEF and volumes (all p-values for interaction <0.07), suggesting that an individual's androgen receptor genotype may substantially modify the relationship between circulating androgens and RV function. For example, higher testosterone levels were associated with lower RVEF in those with two copies of the G risk allele in white men whereas there was no association with RVEF in men who were homozygous for the A allele (figure 3). Effects were unchanged after accounting for LV ejection fraction, suggesting an RV-specific relationship.

\section{Discussion}

Genetic variation in CYP1B1 and the androgen receptor was associated with RV measures (particularly RVEF) in adult men and women respectively without clinical cardiovascular disease. Urinary E2 metabolite levels were associated with RVEF in whites but did not mediate genotype-phenotype relationships in women, whereas androgen receptor SNPs modified the relationship between serum testosterone levels and RV measures in men. In addition to sex, race/ethnicity influenced some of these relationships. The CYP1B1 SNP identified in this healthy cohort has been linked to a SNP associated with disease penetrance in heritable 


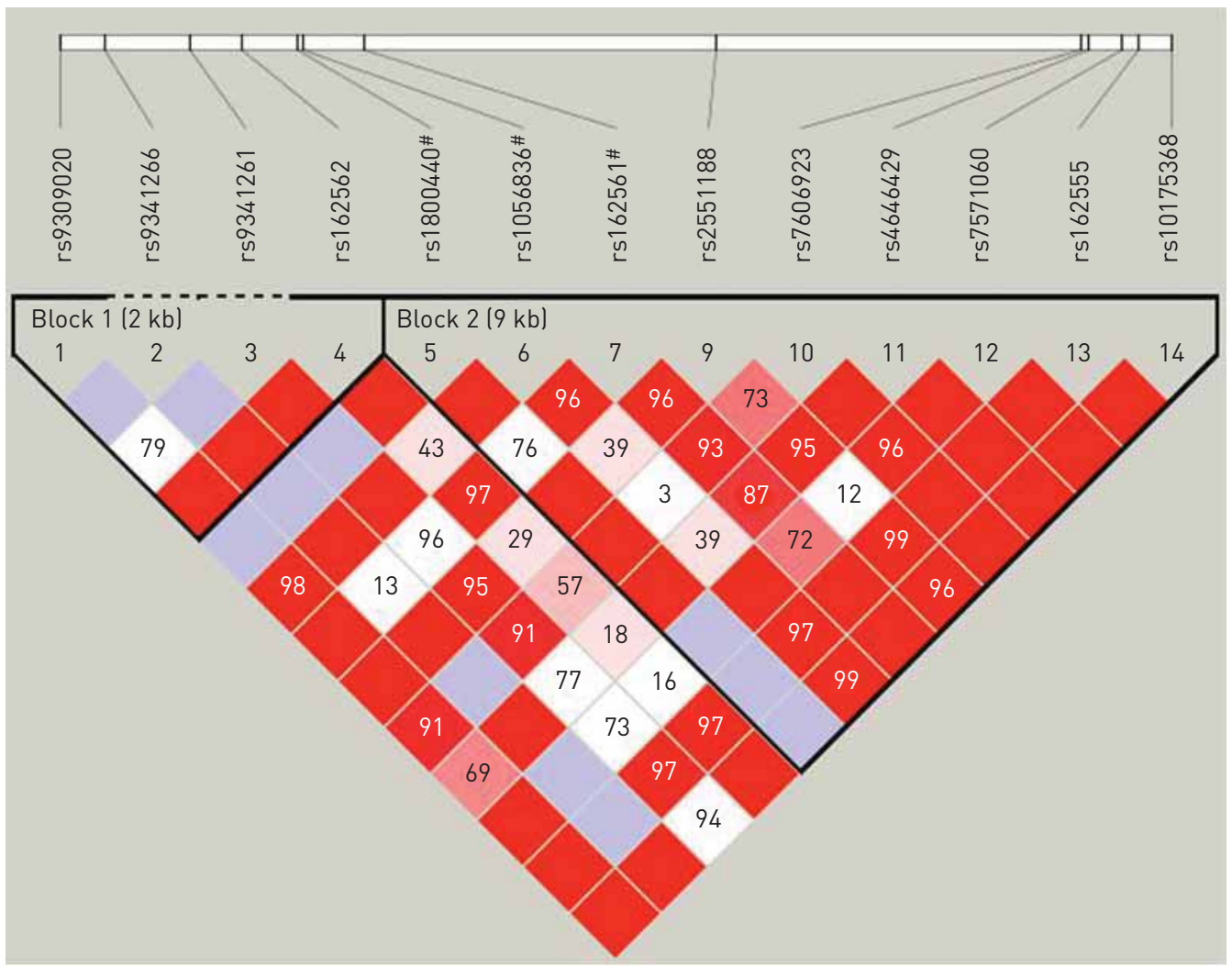

FIGURE 2 Linkage disequilibrium plot for CYP1B1 in African-American women. The white bar represents a chromosome with black lines indicating sequenced single nucleotide polymorphisms (SNPs) in a gene. The colour of a diamond for the intersection of two SNPs indicates likelihood of non-random genetic reassortment such that red regions are most highly correlated (summarised numerically as D' value). ": SNPs of interest. SNP rs162561 was associated with higher right ventricular ejection fraction (RVEF) in African-American women in this study. SNP rs 1800440 has been associated with pulmonary arterial hypertension penetrance in women with bone morphogenetic protein receptor type 2 mutations, which is in tight linkage disequilibrium (LD) with SNP rs162561 ( $D^{\prime}=0.76$ ) [4]. SNP rs1056836 has been associated with breast and lung cancer risk in some studies, also in tight LD with SNP rs162561 $\left(D^{\prime}=0.96\right)$ [29-31]. Block 2 was associated with RVEF in African-Americans (global $p<7.2 \times 10^{-6}$ ), whites (global $p=0.05$ ), and possibly Hispanics (global $p=0.13$ ).

PAH and a SNP tied to oncogenesis in some studies [4, 29-32]. Taken together, these observations support sex- and race-specific genetically determined differences in RV function, and specifically invoke E2 metabolism and androgen signalling as potentially important pathways in RV health and disease.

RVEF is a key determinant of outcome in right heart failure regardless of aetiology, including pulmonary hypertension due to left heart failure as well as advanced lung disease [7-9]. Male sex is associated with worse RV function in heart failure with preserved ejection fraction [13]. In PAH, disease-specific therapy leads to greater improvements in RVEF in women as compared to men, explaining the better outcomes in women [17]. A 3\% increase in RVEF is considered clinically important in PAH, suggesting that the effect estimate seen here between CYP1B1 genotype and RVEF (a 2.0\% increase with each allele) in cardiovascular disease-free individuals could signify relevant genotype-phenotype differences in disease states, especially in rare and extreme phenotypes such as in PAH [33-35]. In men, the per-risk allele increases in mass (approximately $1 \mathrm{~g}$ ) and RVEDV (approximately $6 \mathrm{~mL}$ ) with androgen receptor genotypes are on par with the magnitude of associations between diabetes mellitus and LV mass in MESA and clinically relevant volumetric changes in PAH, respectively $[35,36]$. Since our study was conducted in a cohort free from cardiovascular disease, the genetic determinants of RV structure and function identified here have the potential to play a role in the pathogenesis of RV failure irrespective of cause, although these observations should be confirmed in a diseased cohort.

There were significant associations between CYP1B1 genotype and RVEF in haplotype analysis for AfricanAmerican and white subjects, and genotype-phenotype relationships appeared strongest among AfricanAmerican women. Systemic vascular disease and left heart failure are known to occur more commonly in African-American compared with white subjects even after controlling for socioeconomic factors, and sex hormone levels vary according to race/ethnicity with higher levels of E2 and androgens in African-American men and women compared with white men and women [37]. Observational studies have shown that the 
TABLE 4 Association of urine oestrogen metabolites and RV measures in women, stratified by race for RVEF

\begin{tabular}{|c|c|c|c|c|c|c|c|c|c|c|}
\hline \multirow[t]{2}{*}{ RV measure } & \multirow[t]{2}{*}{ Race/ethnicity } & \multicolumn{3}{|c|}{ Ln(2-hydroxyestrone) } & \multicolumn{3}{|c|}{ Ln(16a-hydroxysterone) } & \multicolumn{3}{|c|}{$\operatorname{Ln}\left(2-\mathrm{OH} / 16-\mathrm{OHE}_{1}\right)$} \\
\hline & & $\boldsymbol{\beta}$ & SE & p-value & $\boldsymbol{\beta}$ & $\mathrm{SE}$ & p-value & $\boldsymbol{\beta}$ & $\mathrm{SE}$ & p-value \\
\hline \multirow[t]{3}{*}{ RVEF } & White & 1.5 & 0.5 & $<0.01$ & 1.3 & 0.4 & $<0.01$ & 0.1 & 0.5 & 0.95 \\
\hline & Hispanic & -0.2 & 0.5 & 0.67 & -1.2 & 0.6 & 0.04 & 0.8 & 0.6 & 0.16 \\
\hline & Chinese-American & 0.3 & 0.6 & 0.60 & 0.4 & 0.7 & 0.63 & 0.3 & 0.7 & 0.66 \\
\hline RV mass & & -0.2 & 0.1 & 0.16 & -0.1 & 0.1 & 0.33 & -0.0 & 0.1 & 0.92 \\
\hline
\end{tabular}

All adjusted for age, race/ethnicity (if no metabolitexrace interaction), height, weight, waist circumference, smoking (pack-years and smoking status), diabetes mellitus, impaired glucose tolerance, systemic hypertension, urine creatinine level, total cholesterol and high-density lipoprotein levels, education level, hormone therapy and respective left ventricle parameter. RVEF: right ventricular ejection fraction; RVEDV: right ventricular end-diastolic volume; 2-OHE: 2-hydroxyestradiol; $16 \alpha-\mathrm{OHE}_{1}$ : $16 \alpha$-hydroxyestrone.

female predominance is even more pronounced in African-American compared with white subjects with $\mathrm{PAH}[18,19]$. Outcomes appear to be poorer in African-American subjects, and we have shown that they respond less well to endothelin receptor antagonism than white subjects with $\mathrm{PAH}$ and that there are baseline differences in RV structure in function that vary by race/ethnicity in health $[16,20,22]$. Whether race/ethnicity-driven differences in sex hormones may be contributing to these epidemiologic observations is not known. Our findings, which directly relate CYP1B1 genotype to RVEF especially in African-American women and show race-based differences in the associations between E2 metabolites and RV function that persist after adjustment for potential confounders such as body size and education, support this concept.

Alterations in E2 metabolism may influence RV structure and function because metabolites appear to have different biochemical effects. Changes in 2-OHE versus $16-\mathrm{OHE}_{1}$ have been linked to phenotypic expression in cardiovascular disease, pulmonary vascular disease, and cancer $[4,6]$. These metabolites have direct and variable mitogenic effects on cardiac cells, but correlations with human ventricular function have not been previously described [38]. The "2-" and "16-" compounds (but not the ratio) had similar relationships with RV measures and did not modify the relationship between CYP1B1 genotype and RV phenotype and there were discordant associations between 2-OHE and 16-OHE1 and RVEF depending on race/ethnicity. Activity and by-products of the cytochrome P450 subfamilies and E2 metabolite balance can be altered acutely during hypoxia, inflammation, with the onset of vascular diseases such as pre-eclampsia and even with dietary changes $[39,40]$. This suggests that the impact of these metabolites on the cardiopulmonary unit is complex and may vary depending on a given woman's race/ethnicity, age, hormonal milieu (endogenous and exogenous) and disease course. As this was a hypothesis-driven candidate gene study, we did not include additional cytochrome mono-oxygenases in our genotype

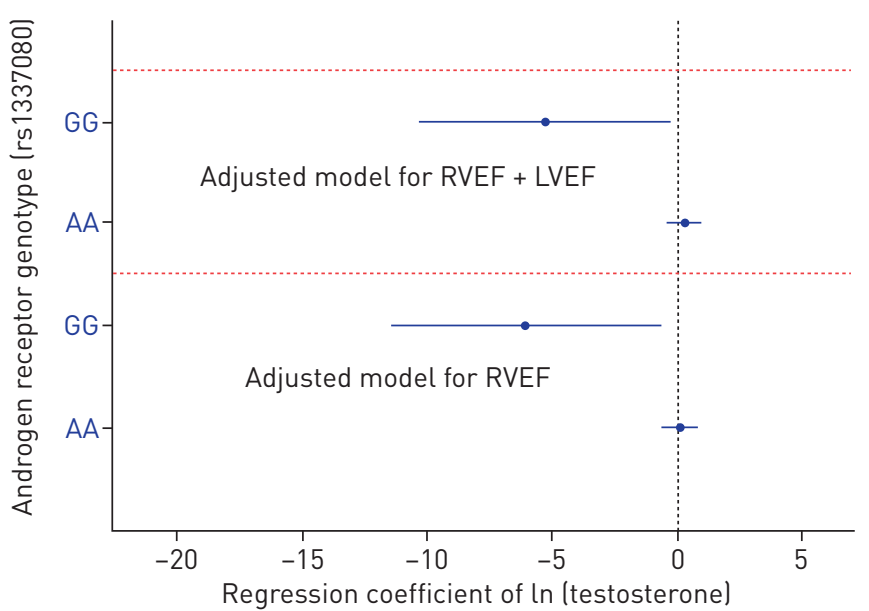

FIGURE 3 Natural log-transformed testosterone parameter estimates with 95\% confidence intervals for right ventricular ejection fraction stratified by androgen receptor genotype for rs1337080 in white men. Models were adjusted for age, height, weight, study site, top three principal components (bottom panel) and left ventricular ejection fraction (top panel). RVEF: right ventricular ejection fraction; LVEF: left ventricular ejection fraction. 
analysis (or other potentially important receptors, such as the G protein-coupled receptor for oestrogen, GPR30) or measure additional metabolite compounds and thus possible relationships between these enzyme products and the RV phenotype are unknown.

The role of androgens in cardiovascular health is controversial because of mixed results with testosterone supplementation in human studies and variable effects in animal models [41]. Our findings in men suggest that circulating testosterone levels could worsen RV function, but that this may be dependent on androgen receptor genotype. Androgen receptors are present in human cardiac myocytes, stimulating hypertrophy with testosterone binding, and higher levels of testosterone are associated with increased fibrosis in the RV under load stress [42, 43]. Testosterone levels have been shown to directly regulate transcription of the androgen receptor during LV hypertrophy in human hearts and androgen receptor expression is associated with metastasis and disease recurrence in carcinoma, but to our knowledge this is the first study linking androgen receptor genotype to RV phenotype [10, 44, 45]. Androgen-receptor interactions may lead to changes in RV morphology via both genomic and non-genomic effects that depend on the androgen, hormone concentration, receptor density of the substrate as well as sex and race of the individual. It has been demonstrated that active sex steroid metabolism occurs in the LV via CYP mono-oxygenases; while this has not been examined in RV tissue, the RV preferentially expresses some of these enzymes, lending support to our observations [10, 11].

Limitations of a cross-sectional, observational study apply here. SNPs representing genetic variation are fixed, and precede both sex hormone metabolism and signalling and RV morphologic changes, fulfilling at least the "temporal" criterion for causality. Smaller subsets had available genotyping and E2 metabolite data as compared to the total MESA-RV cohort; baseline characteristics were similar between the MESA-RV cohort with complete RV measures and covariate data $(\mathrm{n}=4019)$ and the subset with biological samples available $(\mathrm{n}=2761)$ (data not shown). Results were also unchanged when the small number of pre-menopausal women (3\%) were excluded. Ideally 24-h urine collection instead of spot sampling and liquid or gas chromatography-mass spectrometry measurement would have been performed, although the E2 metabolite assay has been employed successfully in other large cohorts, in PAH, and has been validated against mass spectrometry [4, 46, 47]. We failed to show that a shift in E2 metabolite levels mediates the CYP1B1-genotype relationship. This may be due to the assay used (although reproducibility was excellent), lack of measurement of other potentially important metabolites (e.g. products of the 4-hydroxylation pathway), limitations in power as significant interactions necessitated race/ethnicity-stratified analyses, or the inclusion of healthy middle-aged participants.

As this is a population-based cohort, we can only generate hypotheses about the role of the pathways implicated here in RV dysfunction. No large, multi-ethnic studies of robust genotype-phenotype characterisation exist in right heart failure, however, and are only now being established in PAH. There are also no currently available disease-free cohorts with MRI-based measurements of RV structure and function in which we could attempt to replicate our findings. Our study meets several tenets proposed by the National Cancer Institute-National Human Genome Research Institute Working Group on Replication in Gene Association Studies when validation cohorts do not exist [48]. First, pulmonary vascular disease and population-based registries have suggested race- and sex-biases in disease prevalence and outcomes, and numerous preclinical and clinical observations have implicated sex hormone pathways in disease pathogenesis, lending biological insight to our a priori hypotheses [19]. Second, the fact that the genetic variant in CYP1B1 observed here (in a healthy, largely post-menopausal population) is tightly linked to the SNP described by Austin et al. [4] in heritable PAH is noteworthy, as are the demonstrated relationships with serum and urine biomarkers. While we did not find genotype-phenotype associations between other sex hormone signalling and synthesis genes such as oestrogen receptor 1 and 2 and aromatase, there is considerable cross-talk between all of these pathways and this is not to say that these are not relevant in the disease state. Our findings support continued query of the role of E2 metabolism and androgen signalling in determining RV function in women and men respectively with pulmonary hypertension and RV failure $[16,20]$.

\section{Conclusions}

Sex-based differences in right heart function may be explained by alterations in E2 metabolism in women and testosterone-androgen receptor interactions in men in health, and race appears to be an important modifier of some of these relationships.

\section{Acknowledgements}

This manuscript has been reviewed by the MESA Investigators for scientific content and consistency of data interpretation with previous MESA publications. Significant comments have been incorporated prior to submission for publication. The authors thank the other investigators, staff, and participants of MESA for their valuable contributions. A full list of participating MESA Investigators and institutions can be found at www.mesa-nhlbi.org. 


\section{References}

1 Lahm T, Tuder RM, Petrache I. Progress in solving the sex hormone paradox in pulmonary hypertension. Am J Physiol Lung Cell Mol Physiol 2014; 307: L7-L26.

2 Kawut SM, Krowka MJ, Trotter JF, et al. Clinical risk factors for portopulmonary hypertension. Hepatology 2008; 48: 196-203.

3 Roberts KE, Fallon MB, Krowka MJ, et al. Genetic risk factors for portopulmonary hypertension in patients with advanced liver disease. Am J Respir Crit Care Med 2009; 179: 835-842.

4 Austin E, Cogan J, West J, et al. Alterations in estrogen metabolism: Implications for higher penetrance of FPAH in females. Eur Respir J 2009; 34: 1093-1099.

5 Mair KM, Wright AF, Duggan N, et al. Sex-dependent influence of endogenous estrogen in pulmonary hypertension. Am J Respir Crit Care Med 2014; 190: 456-467.

6 White K, Johansen AK, Nilsen M, et al. Activity of the estrogen-metabolizing enzyme cytochrome p450 1b1 influences the development of pulmonary arterial hypertension. Circulation 2012; 126: 1087-1098.

7 Meyer P, Filippatos GS, Ahmed MI, et al. Effects of right ventricular ejection fraction on outcomes in chronic systolic heart failure. Circulation 2010; 121: 252-258.

8 Mohammed SF, Hussain I, Abou Ezzeddine OF, et al. Right ventricular function in heart failure with preserved ejection fraction: A community-based study. Circulation 2014; 130: 2310-2320.

9 Morrison DA, Adcock K, Collins CM, et al. Right ventricular dysfunction and the exercise limitation of chronic obstructive pulmonary disease. J Am Coll Cardiol 1987; 9: 1219-1229.

10 Thum T, Borlak J. Testosterone, cytochrome p450, and cardiac hypertrophy. FASEB J 2002; 16: 1537-1549.

11 Thum T, Borlak J. Gene expression in distinct regions of the heart. Lancet 2000; 355: 979-983.

12 Humbert M, Sitbon O, Chaouat A, et al. Survival in patients with idiopathic, familial, and anorexigen-associated pulmonary arterial hypertension in the modern management era. Circulation 2010; 122: 156-163.

13 Melenovsky V, Hwang SJ, Lin G, et al. Right heart dysfunction in heart failure with preserved ejection fraction. Eur Heart J 2014; 35: 3452-3462.

14 Ventetuolo CE, Ouyang P, Bluemke DA, et al. Sex hormones are associated with right ventricular structure and function: The MESA-Right Ventricle study. Am J Respir Crit Care Med 2011; 183: 659-667.

15 Ventetuolo CE, Praestgaard A, Palevsky HI, et al. Sex and haemodynamics in pulmonary arterial hypertension. Eur Respir J 2014; 43: 523-530.

16 Gabler NB, French B, Strom BL, et al. Race and sex differences in response to endothelin receptor antagonists for pulmonary arterial hypertension. Chest 2012; 141: 20-26.

17 Jacobs W, van de Veerdonk MC, Trip P, et al. The right ventricle explains sex differences in survival in idiopathic pulmonary arterial hypertension. Chest 2014; 145: 1230-1236.

18 D'Alonzo GE, Barst RJ, Ayres SM, et al. Survival in patients with primary pulmonary hypertension. Results from a national prospective registry. Ann Intern Med 1991; 115: 343-349.

19 Badesch DB, Raskob GE, Elliott CG, et al. Pulmonary arterial hypertension: Baseline characteristics from the REVEAL registry. Chest 2010; 137: 376-387.

20 Davis KK, Lilienfeld DE, Doyle RL. Increased mortality in African Americans with idiopathic pulmonary arterial hypertension. J Natl Med Assoc 2008; 100: 69-72.

21 Hyduk A, Croft JB, Ayala C, et al. Pulmonary hypertension surveillance--United States, 1980-2002. MMWR Surveill Summ 2005; 54: 1-28.

22 Kawut SM, Lima JAC, Barr RG, et al. Sex and race differences in right ventricular structure and function: The Multi-Ethnic Study of Atherosclerosis-Right Ventricle Study. Circulation 2011; 123: 2542-2551.

23 Bild DE, Bluemke DA, Burke GL, et al. Multi-Ethnic Study of Atherosclerosis: Objectives and design. Am J Epidemiol 2002; 156: 871-881.

24 Keating BJ, Tischfield S, Murray SS, et al. Concept, design and implementation of a cardiovascular gene-centric 50 k SNP array for large-scale genomic association studies. PLoS One 2008; 3: e3583.

25 Golden SH, Dobs AS, Vaidya D, et al. Endogenous sex hormones and glucose tolerance status in postmenopausal women. J Clin Endocrinol Metab 2007; 92: 1289-1295.

26 Psaty B, Lee M, Savage PJ, et al. Assessing the use of medication in the elderly: Methods and initial experience in the Cardiovascular Health Study: The Cardiovascular Health Study Collaborative Research Group. J Clin Epidemiol 1992; 45: 683-692.

27 Greenland S, Brumback B. An overview of relations among causal modelling methods. Int J Epidemiol 2002; 31 : $1030-1037$.

28 Greenland S. Modeling and variable selection in epidemiologic analysis. Am J Public Health 1989; 79: 340-349.

29 Diergaarde B, Potter JD, Jupe ER, et al. Polymorphisms in genes involved in sex hormone metabolism, estrogen plus progestin hormone therapy use, and risk of postmenopausal breast cancer. Cancer Epidemiol Biomarkers Prev 2008; 17: 1751-1759.

30 Kato I, Cichon M, Yee CL, et al. African American-preponderant single nucleotide polymorphisms (SNPs) and risk of breast cancer. Cancer Epidemiol 2009; 33: 24-30.

31 Wenzlaff AS, Cote ML, Bock CH, et al. CYP1A1 and CYP1B1 polymorphisms and risk of lung cancer among never smokers: A population-based study. Carcinogenesis 2005; 26: 2207-2212.

32 Paracchini V, Raimondi S, Gram IT, et al. Meta- and pooled analyses of the cytochrome p-450 1B1 Val432Leu polymorphism and breast cancer: A HuGE-GSEC review. Am J Epidemiol 2007; 165: 115-125.

33 Roeleveld RJ, Vonk-Noordegraaf A, Marcus JT, et al. Effects of epoprostenol on right ventricular hypertrophy and dilatation in pulmonary hypertension. Chest 2004; 125: 572-579.

34 van de Veerdonk MC, Kind T, Marcus JT, et al. Progressive right ventricular dysfunction in patients with pulmonary arterial hypertension responding to therapy. J Am Coll Cardiol 2011; 58: 2511-2519.

35 Bradlow WM, Hughes ML, Keenan NG, et al. Measuring the heart in pulmonary arterial hypertension (PAH): Implications for trial study size. J Magn Reson Imaging 2010; 31: 117-124.

36 Heckbert SR, Post W, Pearson GDN, et al. Traditional cardiovascular risk factors in relation to left ventricular mass, volume, and systolic function by cardiac magnetic resonance imaging: The Multi-Ethnic Study of Atherosclerosis. J Am Coll Cardiol 2006; 48: 2285-2292.

37 Ouyang P, Vaidya D, Dobs A, et al. Sex hormone levels and subclinical atherosclerosis in postmenopausal women: The Multi-Ethnic Study of Atherosclerosis. Atherosclerosis 2009; 204: 255-261. 
38 Dubey RK, Jackson EK, Gillespie DG, et al. Cytochromes 1a1/1b1- and catechol-o-methyltransferase-derived metabolites mediate estradiol-induced antimitogenesis in human cardiac fibroblast. J Clin Endocrinol Metab 2005; 90: $247-255$.

39 Michnovicz JJ, Bradlow HL. Altered estrogen metabolism and excretion in humans following consumption of indole-3-carbinol. Nutr Cancer 1991; 16: 59-66.

40 Kanasaki K, Palmsten K, Sugimoto H, et al. Deficiency in catechol-o-methyltransferase and 2-methoxyoestradiol is associated with pre-eclampsia. Nature 2008; 453: 1117-1121.

41 Bremner WJ. Testosterone deficiency and replacement in older men. N Engl J Med 2010; 363: 189-191.

42 Marsh JD, Lehmann MH, Ritchie RH, et al. Androgen receptors mediate hypertrophy in cardiac myocytes. Circulation 1998; 98: 256-261.

43 Hemnes AR, Maynard KB, Champion HC, et al. Testosterone negatively regulates right ventricular load stress responses in mice. Pulm Circ 2012; 2: 352-358.

44 He J, Peng R, Yuan Z, et al. Prognostic value of androgen receptor expression in operable triple-negative breast cancer: A retrospective analysis based on a tissue microarray. Med Oncol 2012; 29: 406-410.

45 Tang D, Xu S, Zhang Q, et al. The expression and clinical significance of the androgen receptor and e-cadherin in triple-negative breast cancer. Med Oncol 2012; 29: 526-533.

46 Wellejus A, Olsen A, Tjonneland A, et al. Urinary hydroxyestrogens and breast cancer risk among postmenopausal women: A prospective study. Cancer Epidemiol Biomarkers Prev 2005; 14: 2137-2142.

47 Falk RT, Rossi SC, Fears TR, et al. A new ELISA kit for measuring urinary 2-hydroxyestrone, 16o \pm -hydroxyestrone, and their ratio: Reproducibility, validity, and assay performance after freeze-thaw cycling and preservation by boric acid. Cancer Epidemiol Biomarkers Prev 2000; 9: 81-87.

48 Chanock SJ, Manolio T, Boehnke M, et al. Replicating genotype-phenotype associations. Nature 2007; 447: 655-660. 